\section{Design and Analysis of an Integral Sliding Mode Fault Tolerant Control Scheme}

\author{
Mirza Tariq Hamayun, Christopher Edwards, and Halim Alwi
}

\begin{abstract}
A novel scheme for fault tolerant control is proposed in this paper, in which integral sliding mode ideas are incorporated with control allocation to cope with the total failure of certain actuators, under the assumption that redundancy is available in the system. The proposed scheme uses the effectiveness level of the actuators to redistribute the control signals to healthy actuators without reconfiguring the controller. The effectiveness of the proposed scheme against faults or failures is tested in simulation based on a large transport aircraft model.
\end{abstract}

Index Terms-Fault tolerant control (FTC), linear matrix inequalities (LMIs), integral sliding modes (ISM).

\section{INTRODUCTION}

The challenges of ensuring safety, in critical systems such as aircraft and chemical plant has motivated the need for Fault Tolerant Control (FTC) and has stimulated research in this area. Survey papers such as [1] highlight recent work in the area of FTC and discuss the various application areas. One of the important elements necessary for achieving FTC, is the availability of redundant actuators. This provides increased freedom in terms of controller design to mitigate the effects of faults and failures. For over actuated systems such as aircraft, this requirement is easily satisfied. Although these 'redundant' actuators are often designed for different purposes, in the event of an emergency (such as faults or failures to the primary actuators), they can be used to retain satisfactory performance ${ }^{1}$.

This paper is concerned with the development of fault tolerant controllers for a class of over-actuated linear systems. The redundancy in the over-actuated system will be exploited to achieve tolerance to a specified class of faults/failures, which includes the possibility of total failure to certain primary actuators. Unlike some other schemes in the literature, it is not assumed that the redundancy takes the form of pure replication of certain actuators, and exploits instead the inherent coupling typically present in multi-variable systems. The precise class of total actuator failure which can be accommodated is identified. A novel control scheme is proposed which involves a combination of control allocation and integral sliding mode techniques.

Control Allocation (CA) is one approach which has the capability to manage redundancy in over-actuated systems [3], [4]. In aircraft systems for example, the idea is to design a controller based on a 'virtual' system which provides the desired moments about the center of mass [5]. The virtual control signal is then translated into actual control surface deflections using CA. In terms of the 'virtual' controller design, many methods have been considered in the literature, including LQR [5], and sliding mode control [6], [7], [8]. Classical sliding modes, as employed in [6], [7], [8], consist of two phases: the initial reaching phase (prior to the attainment of a sliding mode), followed by the reduced order sliding motion. During a sliding mode, the closed-loop system is inherently robust to faults in actuators which can be well modeled as matched uncertainty. However this robustness is only achieved during the sliding motion.

M. Hamayun is with the Department of Engineering, University of Leicester, Leicester LE1 7RH, U.K. (email:mth11@le.ac.uk).

The first author would like to acknowledge the support of COMSATS

C. Edwards is with the Department of Engineering, University of Leicester, Leicester LE1 7RH, U.K. (email:chris.edwards@le.ac.uk).

H. Alwi is with the Department of Engineering, University of Leicester, Leicester LE1 7RH, U.K. (email:ha18@le.ac.uk).

${ }^{1}$ An example of this is the Propulsion Controlled Aircraft (PCA) experiments conducted by NASA [2].
To achieve a sliding mode throughout the entire system response, the concept of integral sliding modes (ISM) was proposed in [9], [10] and [11]. In this paper a novel combination of ISM and CA is proposed. This combination allows total failures for a certain subset of the actuators (as well as faults in all actuators) to be accounted for, since sliding mode systems, in common with other traditional feedback systems, are not capable of mitigating total actuator failures without some form of reconfiguration/accommodation. The proposed scheme uses the measured or estimated effectiveness level of the actuators to redistribute the control effort during faults/failures to maintain close to nominal closed-loop performance without reconfiguring the controller. The scheme proposed in this paper has certain advantages compared to [8], which is based on traditional sliding mode control methods. An important advantage is that the analysis of the closed loop system is less complex and less conservative than the work in [8]. Furthermore the stability test proposed in this paper allows a more effective synthesis procedure to be employed to compute the parameters involved in the control law. In fact, a convex representation of the problem can be formulated to allow the use of LMI optimization to synthesize the controller, whereas the approach in [8] requires a 'synthesis-followed-by-analysis' procedure. In this paper the synthesis and analysis is totally integrated.

\section{SYSTEM DESCRIPTION AND PROBLEM FORMULATION}

A LTI system with actuator faults/failures can be represented as

$$
\dot{x}(t)=A x(t)+B W(t) u(t)
$$

where the system and input matrices $A \in \mathbb{R}^{n \times n}, B \in \mathbb{R}^{n \times m}$, and $W(t)=\operatorname{diag}\left\{w_{1}(t), . ., w_{m}(t)\right\}$ is a diagonal matrix. The pair $(A, B)$ is assumed to be controllable. The time varying scalars $w_{1}(t), . ., w_{m}(t)$ model the effectiveness level of the actuators. If $w_{i}(t)=1$, it means that the $i t h$ actuator has no fault and is working perfectly, whereas if $1>w_{i}(t)>0$, an actuator fault is present i.e. the actuator functions with reduced capability. If $w_{i}(t)=0$, actuator $i$ has completely failed and the control input component $u_{i}$ has no effect on the system dynamics. This representation of actuator faults/failures, has been used by many researchers: see for example [12] and [13]. The matrix $W$ will be termed the efficiency matrix indicating the health level of each actuator. Associate with (1) a set of controlled outputs

$$
y_{c}(t)=C x(t)
$$

where $C \in \mathbb{R}^{l \times n}$ and $l<m$. The variables $y_{c}(t)$ are required to respond to desired (external) commands. In terms of "controlling' these outputs only $l$ independent actuators are needed to induce the required closed-loop performance. The remaining $m-l$ actuators constitute redundancy and can be exploited to achieve fault tolerance. In this paper an estimate of the actuator efficiency, $\widehat{W}(t)=\operatorname{diag}\left\{\hat{w}_{1}(t), . ., \hat{w}_{m}(t)\right\}$, where the scalars $0 \leq \hat{w}_{i}(t) \leq 1$, will be used explicitly in the control law. One way to obtain an estimate of the actuator efficiency is by using a measurement of the actual actuator deflection compared to the demand. Such information is typically available in many safety critical systems e.g. passenger aircraft [14]. In other situations $\widehat{W}(t)$ would need to be provided by a Fault Detection and Isolation (FDI) scheme. A sliding mode observer based approach, proposed in [15], can be used to estimate $W(t)$. Because of the properties of sliding mode observers, the error system dynamics collapse in finite time, and do not introduce an extra dynamical error loop which is advantageous. Kalman filter based methods have also been considered to create the estimates $\widehat{W}(t)$, see for example [16]. Whatever method is employed the estimate $\widehat{W}(t)$ will not be perfect and in this paper the difference between 
the actual efficiency matrix $W(t)$, and its estimate $\widehat{W}(t)$ is assumed to satisfy

$$
W(t)=(I-\triangle(t)) \widehat{W}(t)
$$

where $\triangle(t)=\operatorname{diag}\left\{\delta_{1}(t), \ldots \delta_{m}(t)\right\}$. The unknown scalars $\delta_{1}(t), . ., \delta_{m}(t)$ model the level of imperfection in the fault estimation. The effect of this imperfection will be analyzed later in the paper. In this paper a virtual control concept [5] for resolving actuator redundancy will be employed. To this end, the matrix $B$ can be partitioned as:

$$
B=\left[\begin{array}{l}
B_{1} \\
B_{2}
\end{array}\right]
$$

where $B_{1} \in \mathbb{R}^{(n-l) \times m}, B_{2} \in \mathbb{R}^{l \times m}$ of rank $l<m$. Here, as in [8], it is assumed that the elements of $B_{2}$ have large magnitude compared to $\left\|B_{1}\right\|$, so that $B_{2}$ represents the dominant contribution of the control action on the system compared to $B_{1}$. To create this separation, a permutation of the states must usually be undertaken. The virtual control input [5] is defined as

$$
\nu(t):=B_{2} u(t)
$$

where $\nu(t) \in \mathbb{R}^{l}$ can be interpreted as the total control effort produced by the actuators [5]. As in [8], once the partition of $B$ in (4) has been achieved, scale the states so that $B_{2} B_{2}^{T}=I_{l}$. This can be achieved without loss of generality.

The control signal sent to the actuators is

$$
u(t)=B_{2}^{\dagger}(t) \nu(t)
$$

where $B_{2}^{\dagger}(t) \in \mathbb{R}^{m \times l}$ is a right pseudo-inverse of the matrix $B_{2}$. Thus the matrix $B_{2}^{\dagger}(t)$ 'distributes' the virtual control signal to the physical actuators via (6). A generic choice of $B_{2}^{\dagger}(t)$ such that $B_{2} B_{2}^{\dagger}(t)=I_{l}$ is

$$
B_{2}^{\dagger}(t)=\widehat{W}(t) B_{2}^{T}\left(B_{2} \widehat{W}(t) B_{2}^{T}\right)^{-1}
$$

assuming $\operatorname{det}\left(B_{2} \widehat{W}(t) B_{2}^{T}\right) \neq 0$. In the special case when $\widehat{W}(t)=I$, $B_{2}^{\dagger}(t)=B_{2}^{T}$. The overall control structure is given in Figure 1 .

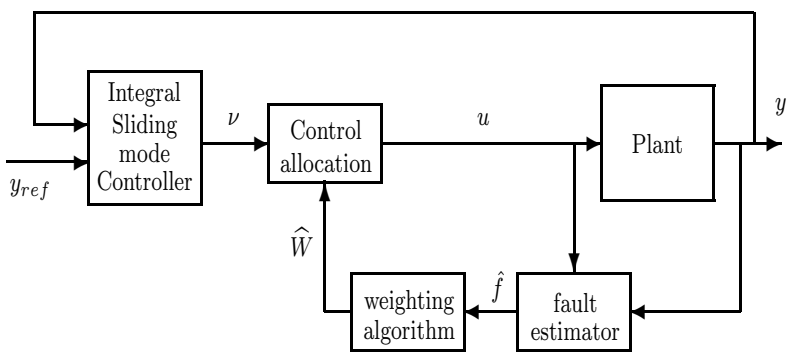

Fig. 1. Schematic of the Overall Control Strategy

Define

$$
\mathcal{W}=\{\left(\hat{w}_{1}, . ., \hat{w}_{m}\right) \in \underbrace{\left[\begin{array}{ll}
0 & 1
\end{array}\right] \times \ldots \times\left[\begin{array}{ll}
0 & 1
\end{array}\right]}_{m \text { times }}: \operatorname{det}\left(B_{2} \widehat{W} B_{2}^{T}\right) \neq 0\}
$$

Because $l<m$, it is possible that $\operatorname{det}\left(B_{2} \widehat{W} B_{2}^{T}\right) \neq 0$ even if up to $m-l$ of the entries $\hat{w}_{i}(t)=0$ in the matrix $\widehat{W}(t)$ : in other words, potentially up to $m-l$ can totally fail and yet $\operatorname{det}\left(B_{2} \widehat{W} B_{2}^{T}\right) \neq 0$. However if more than $m-l$ entries are zero, then $\operatorname{rank}(\widehat{W}(t))<l$ and $\operatorname{det}\left(B_{2} \widehat{W} B_{2}^{T}\right)=0$. The set $\mathcal{W}$ will be shown to constitute the faults/failures for which closed-loop stability can be maintained. Substituting (6) into (1) and using (7) results in

$\dot{x}(t)=A x(t)+\left[\begin{array}{l}B_{1}(I-\triangle(t)) \widehat{W}^{2}(t) B_{2}^{T}\left(B_{2} \widehat{W}(t) B_{2}^{T}\right)^{-1} \\ B_{2}(I-\triangle(t)) \widehat{W}^{2}(t) B_{2}^{T}\left(B_{2} \widehat{W}(t) B_{2}^{T}\right)^{-1}\end{array}\right] \nu(t)$ with

$$
\hat{\nu}(t):=\left(B_{2} \widehat{W}^{2}(t) B_{2}^{T}\right)\left(B_{2} \widehat{W}(t) B_{2}^{T}\right)^{-1} \nu(t)
$$

then (9) can be written as

$$
\dot{x}(t)=A x(t)+\underbrace{\left[\begin{array}{l}
B_{1}(I-\triangle(t)) B_{2}^{+}(t) \\
B_{2}(I-\triangle(t)) B_{2}^{+}(t)
\end{array}\right]}_{\widehat{B}(t)} \hat{\nu}(t)
$$

where

$$
B_{2}^{+}(t):=\widehat{W}^{2}(t) B_{2}^{T}\left(B_{2} \widehat{W}^{2}(t) B_{2}^{T}\right)^{-1}
$$

Notice that $B_{2}^{+}(t)$ is a pseudo inverse of $B_{2}$ since $B_{2} B_{2}^{+}(t)=I$, for all $\widehat{W}(t) \in \mathcal{W}$. Furthermore in the special case when $\widehat{W}(t)=I$, then $B_{2}^{+}(t)=B_{2}^{T}\left(B_{2} B_{2}^{T}\right)^{-1}=B_{2}^{T}$. Whilst the pseudo inverse $B_{2}^{\dagger}(t)$ defined in (7) is used for control allocation, the pseudo inverse $B_{2}^{+}(t)$ defined in (12) plays a significant role in the closed-loop analysis which follows. Using the properties of pseudo inverses detailed in [17], as argued in [8], there exists a scalar $\gamma_{o}$ such that

$$
\left\|B_{2}^{+}(t)\right\|=\left\|\widehat{W}^{2}(t) B_{2}^{T}\left(B_{2} \widehat{W}^{2}(t) B_{2}^{T}\right)^{-1}\right\|<\gamma_{o}
$$

for all $\left(\hat{w}_{1}(t), \ldots, \hat{w}_{m}(t)\right) \in \mathcal{W}$.

In the case when the estimates of the efficiency are perfect (i.e. $\triangle(t)=0$ ), and when there are no faults present (i.e. $\widehat{W}(t)=I$ ), equation (11) simplifies to

$$
\dot{x}(t)=A x(t)+\underbrace{\left[\begin{array}{c}
B_{1} B_{2}^{T} \\
I_{l}
\end{array}\right]}_{B_{\nu}} \nu(t)
$$

since $\left.B_{2}^{+}(t)\right|_{\widehat{W}(t)=I}=B_{2}^{T}$. The nominal fault free equation (14) will be used to design the control scheme. Suppose that by design of the partition in (4), the pair $\left(A, B_{v}\right)$ associated with (14) is controllable, then there exists a state feedback controller $\nu(t)=-F x(t)$, so that the nominal system

$$
\dot{x}(t)=\left(A-B_{\nu} F\right) x(t)
$$

is stable. The state feedback controller can be designed to achieve optimality against some appropriate criteria. The choice of the matrix $F$ will be discussed in the sequel.

\section{Integral Sliding Mode Controller Design}

This section, develops a systematic design procedure for the synthesis of an ISM controller. There are two steps to design an ISM controller, first a sliding surface is designed, and then in the second step, a control law to induce and maintain a sliding motion is created.

\section{A. Integral-type switching surface design}

The integral sliding surface suggested in [11] for the system in (14) associated with the virtual control of input $\nu(t)$ is defined by the set:

$$
\mathcal{S}=\left\{x \in \mathbb{R}^{n}: \quad \sigma(x, t)=0\right\}
$$

where the switching function $\sigma(x, t) \in \mathbb{R}^{l}$ is defined as

$$
\sigma(x, t):=G x(t)-G x\left(t_{0}\right)-G \int_{t_{0}}^{t}\left(A-B_{\nu} F\right) x(\tau) d \tau
$$

and $G \in \mathbb{R}^{l \times n}$ is design freedom. Notice that, at $t=t_{o}$, the switching function $\sigma\left(x\left(t_{0}\right), t_{0}\right)=0$, and hence the reaching phase is eliminated [11]. It can be shown (see for example) [9] that the sliding motion associated with (17) is always nominally governed by $\left(A-B_{\nu} F\right)$ independent of the choice of $G$. Recently an approach was suggested for the selection of $G$ which attempts to ameliorate the effects of unmatched uncertainty [11]. In this paper

$$
G:=B_{2}\left(B^{T} B\right)^{-1} B^{T}
$$


is suggested. Notice that since by definition $B_{\nu}=B B_{2}^{T}$, this choice of $G$ has the property that

$$
G B_{\nu}=B_{2}\left(B^{T} B\right)^{-1} B^{T} B B_{2}^{T}=B_{2} B_{2}^{T}=I_{l}
$$

and so $G$ defined in (18) is a specific choice of left-pseudo inverse of $B_{\nu}$.

To include a tracking facility, the switching function (17) is augmented with a feedforward term $L_{r} r(t)$, where $r(t)$ is the reference signal to track, and

$$
L_{r}:=\left(C\left(B_{\nu} F-A\right)^{-1} B_{\nu}\right)^{-1}
$$

where $C \in \mathbb{R}^{l \times n}$ is associated with the selected controlled outputs in (2). Ultimately the control laws $\nu(t)$ and $u(t)$ will also be augmented with this term. Define a modification to the switching function from (17) as

$$
\sigma(x, t)=G x(t)-G x\left(t_{0}\right)-G \int_{t_{0}}^{t}\left(\left(A-B_{\nu} F\right) x(\tau)+B_{\nu} L_{r} r(\tau)\right) d \tau
$$

where $G$ is defined in (18).

Remark: The following analysis is novel compared to the ISM schemes in [9],[10],[11], since the effects of faults and the actuator redundancy must be taken into account. As a consequence, the analysis in this section is quite distinct compared to the papers cited above because of the incorporation of the ideas from control allocation to exploit the redundancy to ensure sliding can be maintained even in the face of certain total actuator failures.

In order to analyze the sliding motion associated with the surface in (20) and $G$ in (18) in the presence of faults, compute the time derivative of equation (20). It is easy to see

$$
\dot{\sigma}(t)=G \dot{x}(t)-G A x(t)+G B_{\nu} F x(t)-G B_{\nu} L_{r} r(t)
$$

Substituting (11) in (21), and using the fact that $G B_{\nu}=I$, yields

$$
\dot{\sigma}(t)=G \widehat{B} \hat{\nu}(t)+F x(t)-L_{r} r(t)
$$

The equivalent control [18], can be obtained by solving for $\hat{\nu}$ in $\dot{\sigma}(t)=0$ which yields

$$
\hat{\nu}_{e q}(t)=(G \widehat{B})^{-1}\left(-F x(t)+L_{r} r(t)\right)
$$

Substituting the expression in (23) into equation (11) and adding and subtracting $B_{\nu} F x(t)$ yields

$\dot{x}(t)=\left(A-B_{\nu} F\right) x(t)+\left(B_{\nu}-\widehat{B}(G \widehat{B})^{-1}\right) F x(t)+\widehat{B}(G \widehat{B})^{-1} L_{r} r(t)$

where $B_{\nu}$ is defined in (14) and $\widehat{B}$ in (11). Using $G$ as defined in (18), further simplifying equation (24) gives:

$$
\dot{x}(t)=\left(A-B_{\nu} F\right) x(t)+\tilde{B} \tilde{\Phi}(t) F x(t)+\widehat{B}(G \widehat{B})^{-1} L_{r} r(t)
$$

where

$$
\tilde{\Phi}(t):=B_{1} B_{2}^{T}-B_{1}(I-\triangle(t)) B_{2}^{+}(t)\left(B_{2}(I-\triangle(t)) B_{2}^{+}(t)\right)^{-1}
$$

and

$$
\tilde{B}:=\left[\begin{array}{c}
I_{n-l} \\
0
\end{array}\right]
$$

Remark: Notice in the case of perfect knowledge of the actuator efficiency (i.e. $\triangle(t)=0$ ), and when there are no faults in the system (i.e. $\widehat{W}(t)=I)$, the matrices $\left.\widehat{B}\right|_{\widehat{W}(t)=I}=B_{\nu}$ and $\left.B_{2}^{+}(t)\right|_{\widehat{W}(t)=I}=$ $B_{2}^{T}$. Then using the fact that $G B_{\nu}=I$, equation (25) becomes

$$
\dot{x}(t)=\left(A-B_{\nu} F\right) x(t)+B_{\nu} L_{r} r(t)
$$

which is stable by design. Furthermore by choice of $L_{r}$, the controlled output $y_{c}(t) \rightarrow r(t)$. Equation (28) constitutes ideal fault free behavior. However for the generic fault/failure case, the closed-loop stability needs to be proven since the closed-loop system equation (25) depends on matrices $\widehat{W}(t)$ and $\triangle(t)$.

In the presence of faults/failures, the closed-loop system (assuming a sliding motion is maintained) is governed by

$$
\dot{x}(t)=\left(A-B_{\nu} F+\tilde{B} \tilde{\Phi}(t) F\right) x(t)
$$

For the subsequent analysis, define a transfer function matrix

$$
\tilde{G}(s)=F(s I-\tilde{A})^{-1} \tilde{B}
$$

where $\tilde{A}:=A-B_{\nu} F$. By construction, $\tilde{G}(s)$ is stable, and define a scalar

$$
\gamma_{2}=\|\tilde{G}(s)\|_{\infty}
$$

Proposition 1: Assume the effectiveness gain estimate $\widehat{W}(t)$ is sufficiently accurate so that the condition $\triangle_{\max } \gamma_{o}<1$ holds, where $\gamma_{o}$ is defined in (13) and $\|\triangle(t)\|<\triangle_{\max }$. Then during a fault or failure condition, for any $\left(\hat{w}_{1}(t), . ., \hat{w}_{m}(t)\right) \in \mathcal{W}$, the reduced order sliding motion will be stable if:

$$
\frac{\gamma_{2} \gamma_{3}\left(1+\gamma_{o}\right)}{1-\triangle_{\max } \gamma_{o}}<1
$$

where $\gamma_{o}>\left\|B_{2}^{+}(t)\right\|, \gamma_{3}=\left\|B_{1}\right\|$ and $\gamma_{2}$ is as defined in (31).

Proof: The system in (29), which represents the sliding motion can be written as:

$$
\begin{aligned}
& \dot{x}(t)=\tilde{A} x(t)+\tilde{B} \tilde{u}(t) \\
& \tilde{y}(t)=F x(t)
\end{aligned}
$$

where

$$
\tilde{u}(t)=\tilde{\Phi}(t) \tilde{y}
$$

In this form, the differential equation in (29) may be considered to be the closed loop dynamics of the negative feedback interconnection of $\tilde{G}(s)$ and the 'feedback gain' in (35). According to the small gain theorem [19], if

$$
\|\tilde{G}(s)\|_{\infty}\|\tilde{\Phi}(t)\|<1
$$

then (29) will be stable. From (26) it is clear that

$\|\tilde{\Phi}(t)\| \leq\left\|B_{1} B_{2}\right\|+\left\|B_{1}(I-\triangle(t)) B_{2}^{+}(t)\right\|\left\|\left(B_{2}(I-\triangle(t)) B_{2}^{+}(t)\right)^{-1}\right\|$ Using the fact that $\left\|B_{2}\right\|=1, B_{2} B_{2}^{+}(t)=I_{l}$ and also that in general $\left\|(I-X)^{-1}\right\| \leq(1-\|X\|)^{-1}$ if $\|X\|<1$ [20], then

$$
\|\tilde{\Phi}(t)\| \leq\left\|B_{1}\right\|+\left\|B_{1}\right\|\left(1+\triangle_{\max }\right)\left\|B_{2}^{+}(t)\right\|\left(I-\left\|B_{2} \triangle(t) B_{2}^{+}(t)\right\|\right)^{-1}
$$

This is well defined since $\left\|B_{2} \triangle(t) B_{2}^{+}(t)\right\|<\triangle_{\max } \gamma_{o}<1$. Since $\gamma_{o}>\left\|B_{2}^{+}(t)\right\|$ and $\gamma_{3}=\left\|B_{1}\right\|$, inequality (37) becomes

$$
\|\tilde{\Phi}(t)\| \leq \frac{\gamma_{3}\left(1+\gamma_{o}\right)}{1-\triangle_{\max } \gamma_{o}}
$$

Since $\gamma_{2}=\|\tilde{G}(s)\|_{\infty}$, in conjunction with (38), it is clear that if inequality (32) holds, the small gain condition (36) holds, and consequently the system in (29) is stable.

\section{B. Integral Sliding Mode control laws}

Now a sliding mode control law must be designed based on the virtual system (11) with respect to $\hat{\nu}(t)$. The proposed control structure has a form given by:

$$
\hat{\nu}(t)=\hat{\nu}_{l}(t)+\hat{\nu}_{n}(t)
$$

where

$$
\hat{\nu}_{l}(t):=L_{r} r(t)-F x(t)
$$

The scaled unit vector

$$
\hat{\nu}_{n}(t):=\left\{\begin{array}{cl}
-\rho(t, x) \frac{\sigma(x, t)}{\|\sigma(x, t)\|} & \text { if } \sigma(t) \neq 0 \\
0 & \text { otherwise }
\end{array}\right.
$$


where $\rho(t, x)$ is a scalar modulation function to enforce the sliding motion. A suitable choice of $\rho(t, x)$ will be described explicitly in the sequel.

Proposition 2: Suppose that

$$
\|\triangle(t)\| \leq \triangle_{\max }<\frac{1}{\gamma_{o}}
$$

where $\gamma_{o}$ is defined in (13). If $\rho(t, x)$ is chosen as

$$
\rho(t, x)=\frac{\triangle_{\max } \gamma_{o}\left\|\hat{\nu}_{l}\right\|+\eta}{1-\triangle_{\max } \gamma_{o}}
$$

where $\eta$ is a positive scalar, then, the control law proposed in (39) satisfies the so-called reachability condition and sliding on $\mathcal{S}$ in (16) is maintained.

Proof: Substituting (11) in (21) gives

$$
\dot{\sigma}=(G \widehat{B}) \hat{\nu}(t)+F x(t)-L_{r} r(t)
$$

Substituting for $\hat{\nu}(t)$ from (39)-(41) and using the fact that $G \widehat{B}=$ $\left(I-B_{2} \triangle(t) B_{2}^{+}(t)\right)$, gives

$\dot{\sigma}=-\rho \frac{\sigma}{\|\sigma\|}-B_{2} \triangle(t) B_{2}^{+}(t)\left(-F x(t)+L_{r} r(t)-\rho \frac{\sigma}{\|\sigma\|}\right)$ for $\sigma \neq 0$

Consider the candidate Lyapunov function

$$
V(t)=\frac{1}{2} \sigma^{T} \sigma
$$

The time derivative of the Lyapunov function along the trajectories satisfies

$$
\begin{aligned}
\dot{V}= & -\rho\|\sigma\|+\sigma^{T} B_{2} \triangle(t) B_{2}^{+}(t)\left(F x(t)-L_{r} r(t)\right) \\
& +\rho \sigma^{T}\left(B_{2} \triangle(t) B_{2}^{+}(t)\right) \frac{\sigma}{\|\sigma\|} \quad \text { for } \sigma \neq 0
\end{aligned}
$$

and therefore

$$
\begin{aligned}
\dot{V} \leq & -\rho\|\sigma\|+\|\sigma\|\left\|B_{2} \triangle(t) B_{2}^{+}(t)\right\|\|\underbrace{F x(t)-L_{r} r(t)}_{-\hat{\nu}_{l}}\| \\
& +\rho\|\sigma\|\left\|B_{2} \triangle(t) B_{2}^{+}(t)\right\| \\
\leq & -\rho\|\sigma\|+\left(\rho+\left\|\hat{\nu}_{l}\right\|\right)\|\sigma\| \triangle_{\max } \gamma_{o} \\
\leq & -\rho\left(1-\triangle_{\max } \gamma_{o}\right)\|\sigma\|+\left\|\hat{\nu}_{l}\right\|\|\sigma\| \triangle_{\max } \gamma_{o}
\end{aligned}
$$

Substituting for $\rho$ from (43) into (47) gives $\dot{V} \leq-\eta\|\sigma\|$, which is the standard $\eta$-reachability condition [21], and implies that the sliding motion is maintained for all time.

Finally using equations (6), (7) and (10) it follows that the control law is given by

$u(t)=\widehat{W}(t) B_{2}^{T}\left(B_{2} \widehat{W}^{2}(t) B_{2}^{T}\right)^{-1}\left(L_{r} r(t)-F x(t)-\rho \frac{\sigma}{\|\sigma\|}\right)$ if $\sigma \neq 0$

This is the actual control signal which will be sent to the actuators, and depends on the effectiveness levels. The proposed ISM controller (48) can deal with total actuator failures, provided that $\left(\hat{w}_{1}(t), . ., \hat{w}_{m}(t)\right) \in \mathcal{W}$ and the conditions of Proposition 1 are satisfied. The results developed in this section can be summarized in the form of the following theorem:

Theorem 1: The system in (1) is closed-loop stable for any fault/failure combination belonging to $\mathcal{W}$ in (8) under the control law (48), if a feedback gain $F$ can be found such that

$$
\frac{\gamma_{2} \gamma_{3}\left(1+\gamma_{o}\right)}{1-\triangle_{\max } \gamma_{o}}<1
$$

where $\gamma_{2}$ is defined in (31), $\gamma_{3}=\left\|B_{1}\right\|, \gamma_{o}$ satisfies $\gamma_{o} \geq\left\|B_{2}^{+}(t)\right\|$ where $B_{2}^{+}(t)$ is defined in (12), and $\triangle_{\max }$ bounds the relative error in the estimation of the effectiveness gains in (3).

\section{Design of the Controller Gains}

This section demonstrates one of the key advantages of this approach compared to [8]. It will be demonstrated that the stability test in Proposition 1 is amenable to incorporation within a synthesis framework for determining the feedback gain $F$ in (15). For the nominal system (15), the matrix $F$ must be chosen to stabilize $\left(A-B_{\nu} F\right)$. Since $\left(A, B_{\nu}\right)$ is assumed to be controllable, the $\mathrm{LQR}$ formulation adopted here seeks to minimize the cost function

$$
J=\int_{0}^{\infty}\left(x^{T} Q x+u^{T} R u\right) d t
$$

where the matrices $Q$ and $R$ are symmetric positive definite matrices. This problem can be posed as an LMI optimization [22]: Minimize $\operatorname{trace}\left(X^{-1}\right)$ subject to

$$
\left[\begin{array}{cc}
A X+X A^{T}-B_{\nu} Y-Y^{T} B_{\nu}^{T} & (Q X-R Y)^{T} \\
Q X-R Y & -I
\end{array}\right]<0
$$

where $Y:=F X$ with $Y \in \mathbb{R}^{m \times n}$ and $X \in \mathbb{R}^{n \times n}$.

Since, in addition, the small gain stability condition (32) needs to be satisfied, from the Bounded Real Lemma [22], the $\mathcal{L}_{2}$ gain from $\tilde{u}$ to $\tilde{y}$, which in this situation is equal to the $\mathcal{H}_{\infty}$ norm of its transfer matrix $\tilde{G}$, satisfies $\|\tilde{G}\|_{\infty}<\gamma$ iff there exist $X>0$ and $\gamma \geq 0$ such that

$$
\left[\begin{array}{ccc}
A X+X A^{T}-B_{\nu} Y-Y^{T} B_{\nu}^{T} & \tilde{B} & Y^{T} \\
\tilde{B}^{T} & -\gamma^{2} I & 0 \\
Y & 0 & -I
\end{array}\right]<0
$$

where $\tilde{B}$ is defined in (27). Here $\gamma$ is an a-priori fixed scalar gain which may be viewed as a tuning parameter. If

$$
\gamma<\frac{\left(1-\Delta_{\max } \gamma_{o}\right)}{\gamma_{3}\left(1+\gamma_{o}\right)}
$$

then the conditions of Theorem 1 are satisfied and closed loop stability for a fault/failure combination belonging to $\mathcal{W}$ is guaranteed. The overall optimization process is: Minimize $\operatorname{trace}(Z)$ subject to

$$
\left[\begin{array}{cc}
-Z & I_{n} \\
I_{n} & -X
\end{array}\right]<0
$$

together with (49), (50) and (51). The matrix $Z$ is a slack variable which satisfies $Z>X^{-1}$ and therefore $\operatorname{trace}(Z) \geq \operatorname{trace}\left(X^{-1}\right)$. Finally the feedback gain $F$ can be recovered as $F=Y X^{-1}$.

\section{Simulation Results}

The problem of controlling the lateral axis of a large transport aircraft [23] will be used to demonstrate the effectiveness and feasibility of the proposed scheme. A linear model has been obtained around an operating condition of straight and level flight at 263,000 $\mathrm{Kg}, 92.6 \mathrm{~m} / \mathrm{s}$ true airspeed, and at an altitude of $600 \mathrm{~m}$ based on $25.6 \%$ of maximum thrust and at a $20 \mathrm{deg}$ flap position. The states are $[\phi, \beta, r, p]^{T}$, where $\phi$ is roll angle ( $\left.\mathrm{rad}\right), \beta$ is sideslip angle ( $\left.\mathrm{rad}\right), r$ is yaw rate $(\mathrm{rad} / \mathrm{sec})$, and $p$ is roll rate $(\mathrm{rad} / \mathrm{sec})$. The controlled outputs are $[\beta, \phi]$, which means $l=2$. The available control surfaces are $\delta=\left[\delta_{a}, \delta_{r}, \delta_{e p r}\right]^{T}$, which represent anti-symmetric aileron deflection (rad), rudder deflection (rad) and differential aggregated engine pressure ratios (EPR). Note in this example $m=3$ while $l=2$, and so in theory only two control inputs would be required to force the controlled outputs to follow a commanded trajectory. Here the fact that three control inputs can be manipulated, indicates the existence of redundancy in the system which can be exploited to achieve fault tolerance. The ordering of the states ensures $\left\|B_{1}\right\|<<\left\|B_{2}\right\|$. After scaling the states to ensure $B_{2} B_{2}^{T}=I_{l}$ the state-space representation is

$$
A=\left[\begin{array}{rrrr}
0 & 0 & 0.0084 & 0.3334 \\
0.1055 & -0.0999 & -0.3170 & 0.0538 \\
-0.0059 & 0.5617 & -0.1856 & -0.1796 \\
0.0008 & -4.8828 & 0.2154 & -1.0789
\end{array}\right]
$$




$$
B=\left[\begin{array}{rrr}
0 & 0 & 0 \\
0 & 0.0174 & -0.0010 \\
\hline-0.1459 & -0.7584 & -0.6352 \\
-0.9387 & 0.3089 & -0.1531
\end{array}\right]\left\{B_{1}\right.
$$

In a fault free scenario, i.e in normal flight, the primary control surfaces for $\phi$ and $\beta$ tracking are the ailerons and rudder respectively; however the engine thrust can be used as redundancy for both surfaces. Based on these assumptions, using a numerical search, it was found that a suitable bound for the scalar in (13) is $\gamma_{o}=3.2020$. Consequently the maximum error in efficiency estimation which can be tolerated is $\frac{1}{3.2020}=0.3123$. Here $\triangle_{\max }=0.25$ is chosen to satisfy this requirement which implies an upper bound on the relative error in $\widehat{W}$ of $25 \%$. It can be easily verified that $\gamma_{3}=\left\|B_{1}\right\|=0.0174$. It can be shown that in order to satisfy the requirements of Theorem 1 , the scalar $\gamma_{2}=\|\tilde{G}(s)\|_{\infty}$ must satisfy $\gamma_{2}<\frac{1}{0.3671}=2.7240$. The nominal state feedback controller gain $F$ associated with (15) has been designed using the LMI approach proposed in Section III-C. The nominal performance design matrices $Q$ and $R$ in (49) have been chosen as $Q=\operatorname{diag}\{1,1,10,10\}$ and $R=\operatorname{diag}\{7,7\}$ respectively. The choice of $\gamma=14$ in (51) results in $\gamma_{2}=2.1061<2.7240$ and it can be verified that

$$
\frac{\gamma_{2} \gamma_{3}\left(1+\gamma_{o}\right)}{1-\triangle_{\max } \gamma_{o}}=0.7719<1
$$

and therefore the condition of Theorem 1 is satisfied ${ }^{2}$. Consequently the closed-loop stability of the system for any combination of faults $\left(\hat{w}_{1}, . ., \hat{w}_{3}\right) \in \mathcal{W}$ is ensured.

In the simulations which follow the linear aircraft model undertakes a turning manoeuvre, where the reference command requests a change in $\phi$ to $25 \mathrm{deg}$ during the period of time $60-90 \mathrm{sec}$, whilst a $0 \mathrm{deg}$ reference command is applied to $\beta$ throughout. In some applications the discontinuous controller can be applied directly. In this case it is not possible, and the discontinuity in (41) is smoothed using the sigmoidal approximation [21], $\frac{\sigma}{\|\sigma\|+\delta_{o}}$ where the value of the positive scalar $\delta_{o}$ is chosen to be 0.001 . An ideal sliding motion will not be obtained in this situation, and instead the switching function $\sigma(t)$ will be forced into a boundary layer around $\mathcal{S}$. This can be made arbitrarily small by selecting $\delta_{o}$ sufficiently small. The loss of ideal sliding results in another (exogenous) signal, depending on $\sigma(t)$, impacting on (25). However the stability analysis of the sliding motion associated with (29) is still valid. Figure 2 and Figure 3 show various levels of aileron faults (from $0 \%-100 \%$ ) each occurring at 80 -sec in $15 \%$ increments. It can be seen that the CA systematically redistributes the control signals to the rudder and the engines, while maintaining the same level of tracking performance as in the fault free condition.

Figure 4 and Figure 5 show the tracking performance of the states and the control surface deflections, when a rudder jam occurs at 80sec $-2 \mathrm{deg}$. In the failure case the control signal sent to the rudder is shutoff as the effectiveness level vanishes. Although not shown, in all the simulated scenarios, a sliding mode exists for the whole of the simulation period.

\section{CONCLUSION}

A novel Integral Sliding Mode fault tolerant control scheme has been proposed in this paper. To handle total actuator failures, integral sliding mode ideas are incorporated into a control allocation framework, which has the capability to redistribute the control effort

\footnotetext{
${ }^{2}$ Choosing $\gamma=2.7240$ in (51) will guarantee that $\gamma_{2}<2.7240$ but will unnecessarily limit the nominal performance of the controller. Because of the conservatism resulting from the common solution to (49)-(51) a larger value of $\gamma$ has been used during the synthesis but this still results in $\gamma_{2}<2.7240$, which satisfies the requirements of Theorem 1 .
}

among the healthy redundant actuators automatically in the case of faults/failures without reconfiguring the controller. The estimation of the actuator effectiveness levels is a key source of information for the control allocation scheme. A new stability analysis ensures closedloop stability of the system for a certain level of mismatch between the actual and the estimated fault and in fact the synthesis problem can be posed as a convex optimization in terms of the parameters of the controller. The efficiency of the proposed fault tolerant scheme has been demonstrated through simulation based on fault scenarios in a large transport aircraft.

\section{REFERENCES}

[1] Y. Zhang and J. Jiang, "Bibliographical review on reconfigurable faulttolerant control systems," Annual Reviews in Control, vol. 32, no. 2, pp. 229-252, 2008.

[2] T. Tucker, Touchdown: The development of propulsion controlled aircraft at NASA Dryden. Monographs in Aerospace History, no 16, 1999.

[3] J. Bošković and R. Mehra, "Control allocation in overactuated aircraft under position and rate limiting," in American Control Conference, vol. 1, Anchorage, AK, 2002, pp. 791-796.

[4] J. Davidson, F. Lallman, and W. Bundick, "Real-time adaptive control allocation applied to a high performance aircraft," in 5th SIAM Conference on Control and Its Application, San Diego, CA, 2001.

[5] O. Härkegård and S. T. Glad, "Resolving actuator redundancy - optimal control vs. control allocation," Automatica, vol. 41, no. 1, pp. 137-144, 2005.

[6] R. Hess and S. Wells, "Sliding mode control applied to reconfigurable flight control design," Journal of Guidance, Control and Dynamics, vol. 26, no. 3, pp. 452-462, 2003.

[7] Y. Shtessel, J. Buffington, and S. Banda, "Tailless aircraft flight control using multiple time scale re-configurable sliding modes," IEEE Transactions on Control Systems Technology, vol. 10, no. 2, 2002.

[8] H. Alwi and C. Edwards, "Fault tolerant control using sliding modes with on-line control allocation," Automatica, vol. 44, no. 7, pp. 18591866, 2008.

[9] V. Utkin and J. Shi, "Integral sliding mode in systems operating under uncertainty conditions," in 35th IEEE Conference on Decision and Control, 1996, pp. 4591-4596.

[10] W. Cao and J. Xu, "Nonlinear integral type sliding surface for both matched and unmatched uncertain systems," IEEE Transactions on Automatic Control, vol. 49, no. 8, pp. 1355-1360, 2004.

[11] F. Castanos and L. Fridman, "Analysis and design of integral sliding manifolds for systems with unmatched perturbations," IEEE Transactions on Automatic Control, vol. 51, no. 5, pp. 853-858, 2006.

[12] Y. Zhang and J. Jiang, "Fault tolerant control system design with explicit consideration of performance degradation," IEEE Transactions on Aerospace and Electronic Systems, vol. 39, no. 3, pp. 838-848, 2003.

[13] G. Tao, S. M. Joshi, and X. Ma, "Adaptive state feedback and tracking control of systems with actuator failures," IEEE Transactions On Automatic Control, vol. 46, no. 1, pp. 78-95, 2001.

[14] D. Brière and P. Traverse, "Airbus A320/A330/A340 electrical flight controls: A family of fault-tolerant systems." Digest of Papers FTCS-23 The Twenty-Third International Symposium on Fault-Tolerant Computing, pp. 616-623, 1993.

[15] H. Alwi and C. Edwards, "Sliding mode FTC with on-line control allocation," in 45th IEEE CDC, San Diego, CA, 2006, pp. 775-795.

[16] N. Wu, Y. Zhang, and K. Zhou, "Detection, estimation, and accommodation of loss of control effectiveness," International Journal of Adaptive Control and Signal Processing, vol. 14, no. 7, pp. 775-795, 2000.

[17] G. Stewart, "On scaled projections and pseudoinverses," Linear Algebra and its Applications, vol. 112, pp. 189-193, 1989.

[18] V. Utkin, J. Guldner, and J. Shi, Sliding Mode control in Electromechanical Systems. London, UK: Taylor and Francis, 1999.

[19] H. Khalil, Nonlinear Systems. Englewood Cliffs NJ.: Prentice Hall, 1992.

[20] R. A. Horn and C. R. Johnson, Matrix Analysis. Cambridge, UK: Cambridge University Press, 1990.

[21] C. Edwards and S. Spurgeon, Sliding Mode control, Theory and Applications. New York: Taylor and Francis, 1998.

[22] S. Boyd, L. Ghaoui, E. Feron, and V. Balakrishnan, Linear Matrix Inequilities in System and Control Theory, Philadelphia, PA, SIAM 1994.

[23] C. Edwards, T. Lombaerts, and H. Smaili, Fault Tolerant Flight Control: A Benchmark Challenge. Berlin Heidelberg: Springer-Verlag, 2010, vol. 399. 

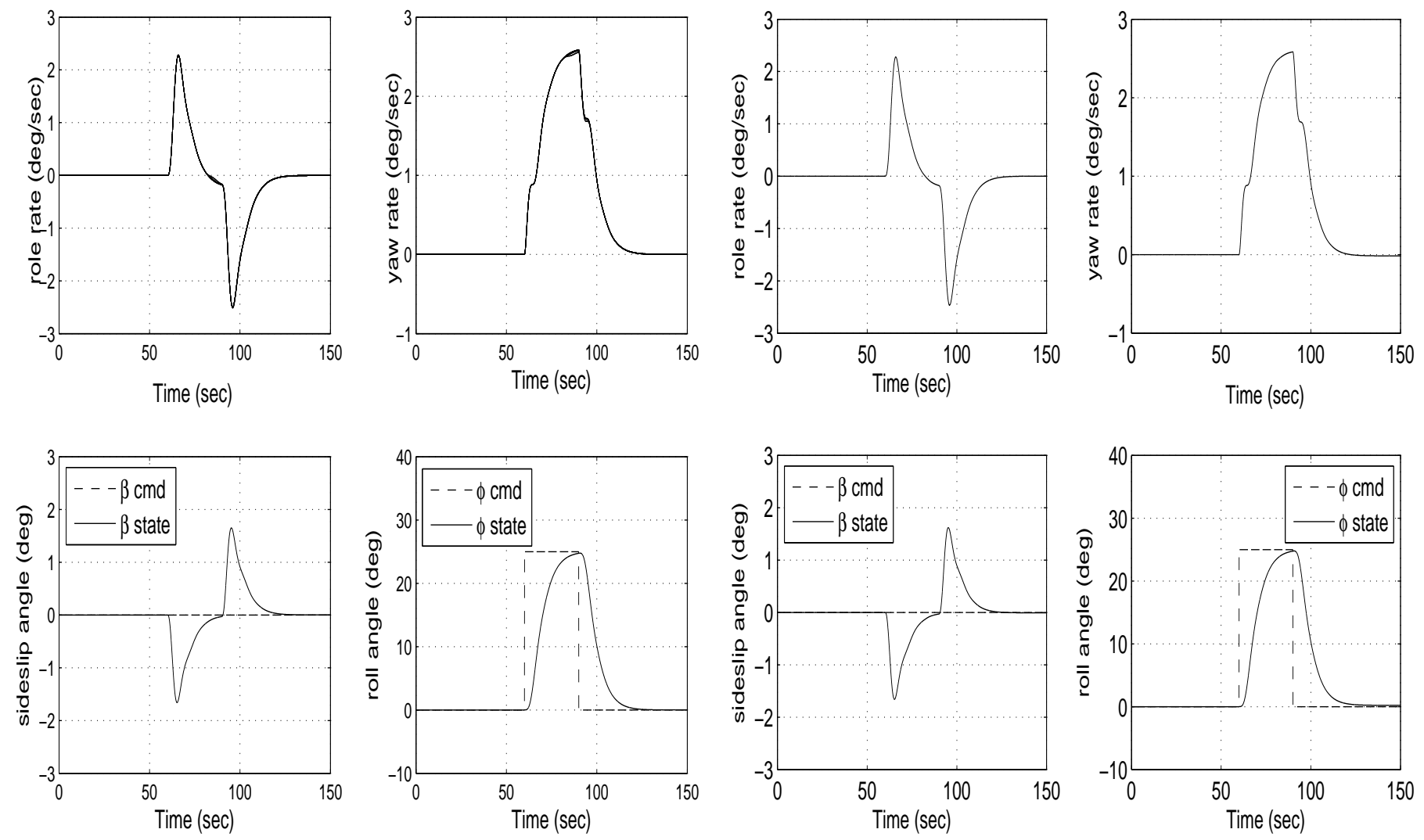

Fig. 2. Aileron-fault: plant states

Fig. 4. Rudder offset-jam: plant states
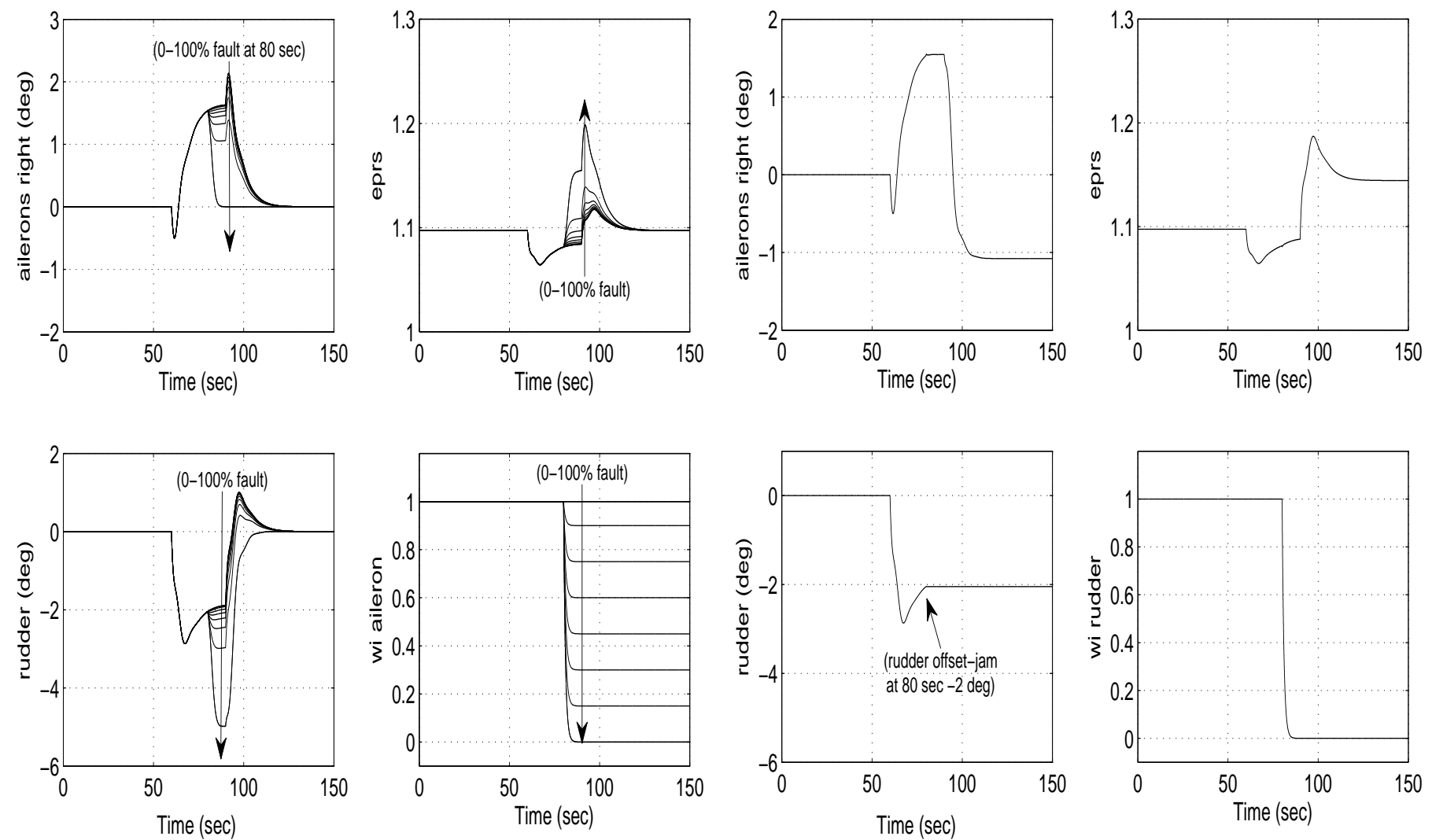

Fig. 3. Aileron-fault: actuator deflections
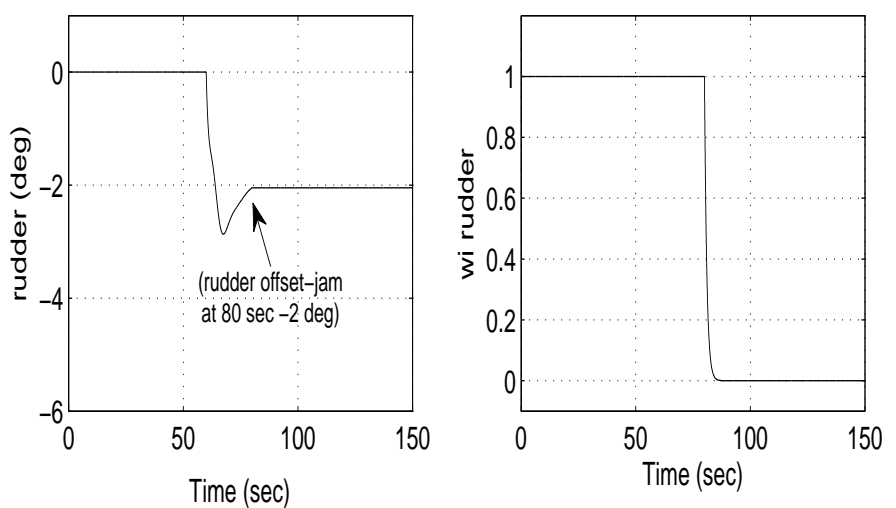

Fig. 5. Rudder offset-jam: actuator deflections 